\title{
System Construction of Ecological Landscape Evaluation Based on Simulation Technology of Computer Vision
}

\author{
Sirong Liu, Song $\mathrm{Xu}$ \\ School of Civil Engineering and Building Academy, Hubei University of Technology, Wuhan, China \\ liusirong1@yeah.net
}

Keywords: polymerization method; landscape index; evenness; fragmentation; network analysis

\begin{abstract}
In the world scope, the pace of urbanization constantly speeds up, and gradual the migration population from city to urban have negative ecological effect on the environment of coastal cities. But coastal areas link up the land and Marine ecological system,which is an important part of the wildlife habitats and traditional geographical landscape. The expansion of urban areas is more and more threatening to coastal landscape, natural monuments and coastal agriculture. Based on an overall landscape method, according to the sustainable development theory, under the ensurence of the agriculture and social economic problems, for the ecological network and heritage protection from the angle of theory and practice, the paper provids a natural resource management and reduce ecological influence caused by human beings on effective information and solution, which provides the open green space for the growing coastal city residents.
\end{abstract}

\section{Introduction}

Scientific and effective research of the diversity of coastal ecological cultural, first of all is to understand the concept of the landscape structure and landscape change; Then, through application the land intensively, keeping small natural patch and corridor in the developed area, arranging human activity along the main boundary to make the landscape to the ecological balance. Landscape method is the use of not only the natural science knowledge, but also the social science knowledge to manage ecological system[1].

Only by reaching the detailed, thorough understanding of the development of ecological system under the influence of human beings, and application the method of landscape and ecological system correlation technique, can the government ensure the decision carried out scientifically[2].

\section{The factors that affects the diversity of coastal overall ecology}

A. The influence of climate on diversity of ecology

In recent years, systematic and deeply research of the diversity of ecology on what the climate change bringabout biological species vulnerability, the natural and artificial adaption to climate change and other aspects, work out the countermeasures of systematically analysis the diversity of ecology to adapt to climate change, including the adaption of technology and effect of diversity of ecology on climate change. the technology including planning and management of nature reserve, artificial migration of species, setting up germplasm gene pool, the development of the ecology system's adaptability, disaster forecasting, as well as to analyze the effect which countermeasures will produce, etc.

B. The interference of human beings

Since the 1990 s, the decay of agriculture department is also become an another important factor of depravation of coastal environment. The coastal areas is faced with the threat of the raising value of the land.

C. Natural ecosystem repairment

Natural ecological system space recovery takes the form of species composition of ecological memory. The interaction between species, restructuring, makeing ecosystem recovery becomes possible. Ecological memory can be seen everywhere in the abandoned site and sabotage place. The performance of whole process is that the existing static memory should particularly added to 
dynamic memory, such as ecology cultivated land is a part of ecology system management, and dynamic succession of natural interference condition is similarly imitated in landscape.

Zasada puts forward that the effective protection of nature is not to protect a piece of territory in isolation, butbring territory into the overall space nature protection system through the policy. Although we pay attention to space ecosystem recovery of territory accord with the landscape planning idea, the result of action is decided by the interaction of the natural and artificial factors.

\section{The method of landscape ecology analysis on coastal overall diversity of ecology culture}

The main function of landscape ecology analysis is to protect and improve the biology diversity. If you want to know whether goals of natural resource protection and recovery is consistent with biological diversity, bringing the introduction of various statistical methods in evaluating them reasonably. We can put the Landscape polymerization method (Landscape Cohesion, LC) and ecological scale extrapolation Landscape index method (Ecologically Scaled Landscape Indices) and space sustainable standard (spaital sustainability standards) together. The following landscape index method is what the paper used:

(1)Species diversity index

Formula of Shannon - Wiener index is[3]

$$
H=-\sum P_{i} \operatorname{In} P_{i}
$$

$\mathrm{H}$ is population diversity index, $P_{i}$ is the rate of the first $\mathrm{i}$ of individual of the study. That is the ratio of first I species of individual number and the total individual all species, and In is the natural logarithm.

(2)The uniformity index

Uniformity is described distribution uniformity degree of the landscape in different types of landscape. Formula of Pielou index is[4]

$$
E=H^{\prime} / H^{\prime}{ }_{\text {max }}=H^{\prime} / \text { InS }
$$

E is uniformity index, $H^{\prime}$ is the diversity index value, $H^{\prime}{ }_{\text {max }}$ is the biggest diversity index value, $\mathrm{S}$ is number of species. The larger the value of homogeneous degree is, the more homogeneous the composition distribution will be.

(3)The fractal dimension

It is used to measure how the shape of patches affect the ecological process of internal patches which is the shape of patch. It is 2 times of the regression coefficient. Namely:; D is fractal dimension, $\mathrm{k}$ is the regression coefficient between Plaque area and perimeter , 1 is the circumference of the patches, $\mathrm{s}$ is the same plaque area, $\mathrm{C}$ is constant.

(4)The heterogeneity index

Heterogeneity index ( HT ) is research on indeterminateness, the more abundant element type is, the higher the fragmentation degree is, the more indeterminate information contained in a landscape system. The size of the index directly reflect the number of landscape elements and proportion changes of landscape element. constitute of Landscape elements is simplex,and landscape is homogeneous,then then the heterogeneity index was 0 ; case is consist of two or more than two elements. If all landscape types is equal in proportion, the highest the diversity is; the more different proportion of each type of landscape, the smaller landscape diversity is. Heterogeneity index is calculated application formula[5]:

$$
H T=-\sum P_{i} \operatorname{In} P_{i}
$$

$P_{i}$ is the proportion of a landscape of land use unit accounted for of the total area landscape.

(5)The decision dominance

It used to measure degree of one or several types of landscape control landscape of the landscape structure, by introducing maximum of heterogeneity index, normalized, as is former correction: method for advantage degree calculation is[6] 
$D_{m}=I_{n}(n)-H T$

It is dominance, $\mathrm{n}$ is landscape unit type, $I n(n)$ is the maximum value of HT. $D_{m}$ is small means landscape consists of several roughly equal proportion type, value is larger, on the contrary, which means landscape is controlled by only one or a small number of several types. If the study population is above $5 \%$ of the total number of species for the investigation, we set it the dominant species.

(6) The fragmentation

The value of fragmentation reflects the situation of species' breeding, migration, diffusion, protection.

Formula for Fragmentation $C=\sum n_{i} / A, \sum n_{i}$ is the number of patches in all landscape types in the landscape, A is total area for landscape[7].

TABLE I. The area of patches and number of application types of different lands in coastal areas in 1993

\begin{tabular}{|c|l|l|l|l|l|l|l|}
\hline $\begin{array}{c}\text { Types of land } \\
\text { application } \\
\text { area (m } \mathbf{~}^{2}\end{array}$ & $\begin{array}{c}\text { Area } \\
\text { (million } \mathbf{~ m}^{2} \text { ) }\end{array}$ & ratio & $\begin{array}{c}\text { plaque } \\
\text { number }\end{array}$ & $\begin{array}{l}\text { patch } \\
\text { ratio }\end{array}$ & $\begin{array}{l}\text { average } \\
\text { patch } \\
\text { (million m } \mathbf{~}^{\text {) }}\end{array}$ & $\begin{array}{l}\text { average patch } \\
\text { perimeter (m) }\end{array}$ & $\begin{array}{c}\text { Average patch } \\
\text { fractal dimension D }\end{array}$ \\
\hline Village & 109.5 & $0.78 \%$ & 24 & $24.00 \%$ & 4.6 & 984.06 & 1.03 \\
\hline living & 42 & $0.30 \%$ & 14 & $14.00 \%$ & 3.0 & 840.40 & 1.04 \\
\hline public & 106 & $0.6 \%$ & 41 & $41.00 \%$ & 1.8 & 625.39 & 1.03 \\
\hline industry & 72.8 & $0.52 \%$ & 5 & $5.00 \%$ & 21.2 & 1884.90 & 1.02 \\
\hline storage & 11.3 & $0.08 \%$ & 7 & $7.00 \%$ & 1.6 & 597.03 & 1.03 \\
\hline particular & 46.6 & $0.33 \%$ & 9 & $9.00 \%$ & 5.2 & 962.63 & 1.02 \\
\hline
\end{tabular}

TABLE II. The area of patches and number of application types of different lands in coastal areas in 2011

\begin{tabular}{|c|c|c|c|c|c|c|c|}
\hline $\begin{array}{l}\text { Types of land } \\
\text { application area }\left(\mathrm{m}^{2}\right)\end{array}$ & $\begin{array}{c}\text { Area } \\
\left(\text { million } \mathbf{m}^{2}\right)\end{array}$ & ratio & $\begin{array}{l}\text { plaque } \\
\text { number }\end{array}$ & $\begin{array}{c}\text { patch } \\
\text { ratio }\end{array}$ & $\begin{array}{c}\text { average patch } \\
\text { area }\left(\text { million } \mathbf{m}^{2}\right)\end{array}$ & $\begin{array}{c}\text { Average } \\
\text { patch peri } \\
\text { meter (m) }\end{array}$ & $\begin{array}{l}\text { average patch } \\
\text { fractal dimension D }\end{array}$ \\
\hline Village & 648.7 & $4.64 \%$ & 91 & $52.30 \%$ & 7.1 & 1154 & 1.03 \\
\hline living & 86.4 & $0.62 \%$ & 17 & $9.77 \%$ & 5.1 & 832 & 1.04 \\
\hline public & 178.7 & $1.28 \%$ & 46 & $26.44 \%$ & 3.9 & 1022 & 1.06 \\
\hline industry & 147.6 & $1.06 \%$ & 10 & $5.75 \%$ & 14.8 & 1675 & 1.04 \\
\hline storage & 17.7 & $0.13 \%$ & 7 & $4.02 \%$ & 2.5 & 659 & 1.02 \\
\hline particular & 23.1 & $0.16 \%$ & 3 & $1.72 \%$ & 7.7 & 1173 & 1.02 \\
\hline Village & 12870.8 & $92.11 \%$ & & & & & \\
\hline total & 13973 & $100.00 \%$ & & & & & \\
\hline
\end{tabular}

\section{The application of landscape ecological analysis and the rule model}

By application landscape ecological analysis and rule model,we can realize three purpose as follows[8,9]: (1) identify practical ecological system present; (2) evaluate wether the network is able to improve the present situation according to the designed ; (3) identify the opportunities of flash ecological network and spread evaluation target species have habitat conditions by taking a region or ecological system don't chosen as the basis.

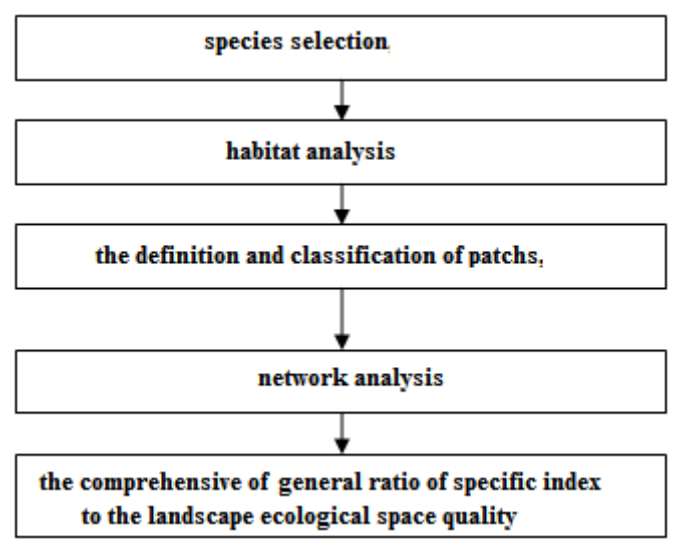

Figure 1. The landscape ecological analysis and the rules of procedure chart

A. Species selection

The systems approach of Choosing species is to make a matrix which including the level of diffusion distance (for example: $<100 \mathrm{~m}, 100-1000 \mathrm{~m}, 1000-10000 \mathrm{~m}$ ) and the level of nest domain range, and then filling the matrix by choosing representative species in different groupand habitat. 
In addition,we can also draw the ecological section to replace real species or choose species which have special value.

B. Habitat analysis

Habitat evaluation mainly divided into two parts:the overall and the local. The standard basis of species habitat suitability, and evaluate different location and areas esynthetically. Habitat analysis can be divided into two categories: one is the analysis on regional scale, through setting up the habitat suitability mode of general information the other one is found the mathematical model based on the relationship of research points or quadrat construct species and habitat.

To assign every factor assignment to qualitative standard factor, and carries on the fuzzy comprehensive analysis to obtain the habitat suitability:

(1)Habitat index(HSI) : to determine the value of habitat factor as $0-1$, then find out the index of habitat which is the Ensemble average of each environment . The formula is[10]:

$$
H=\sqrt[3]{V_{1} \times V_{2} \times V_{3}}
$$

HIS's important significance is presenting repeated assessment procedure, expressing influence of environment factors on the species simply, and offforing a characteristic index of particular environment for evaluation on management plan.

(2) Habitat evaluation program (HEP): This procedure is mainly used to evaluate the feedback information of the effects of environmental conditions on the species and habitat change of species. The bird species and quantity in Coastal areas of Guangzhou is shown below:

TABLE III. The constitution of bird species and quantity in Coastal areas of Guangzhou

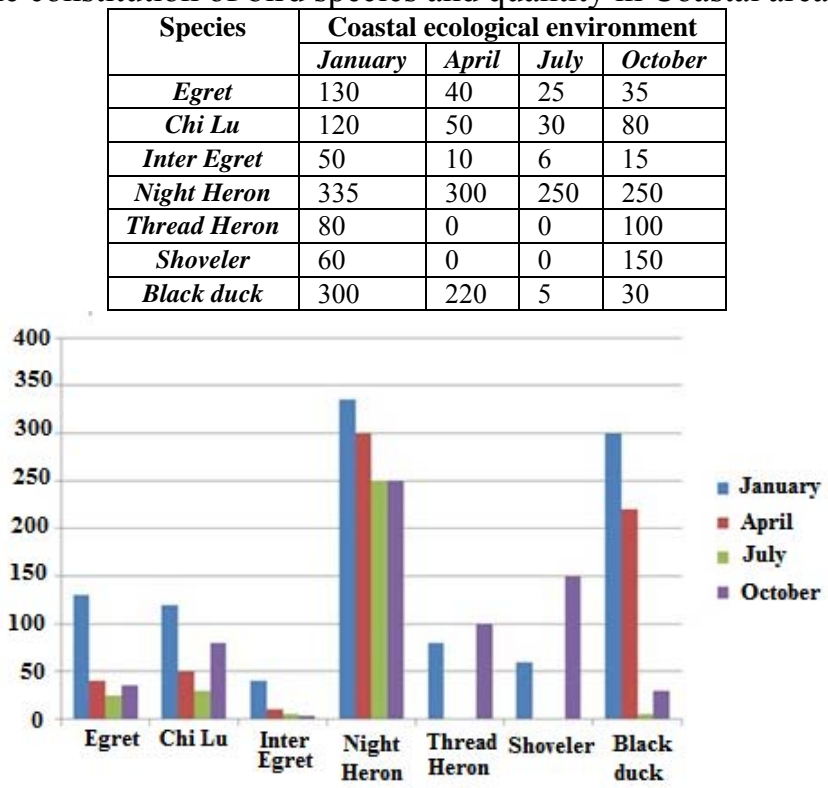

Figure 2. The rectangular diagram of species and quantity of the birds in Guangzhou coastal areas

C. Definition and classification of the of patches

From the habitat diagram,we can see every habitat fragment has its bearing capacity. the patches which is separated less than 1.5 times of individual nest area radius of the distance can be combined to the population in patches. The population patches was divided into: undersized patch ( small capacity $<1$ ); the minimum viable population of patch, which is large enough to accommodate a minimum viable population patch; key patch, which is too small for a minimum viable population patch but capable of accommodating a key population; small patch ( capacity $>1$ ), which cannot reach the demand of the key patch.

D. Network analysis

If patches distance is near enough to form a heterogeneous population habitat network, we consider that they can form a habitat network in specific diffusion range. If the minimum live heterogeneous population (MVMP) can meet the standard, then habitat network is sustainable, and one of the heterogeneous population border live in that can survive. Explanation is as follows: if the network capacity in the essential value of 1-5 times the room, it can be calibrated for: live (in 100 years the possibility of extinction estimated at $1 \%-5 \%$ ). 
E. The general ratio analysis from the concrete index to the landscape ecological space quality

When the ratio is 1 , which means that network in heterogeneous population is minimum living population. When ratio greater than 1 means that species is sustainable, and when it less than 1 means unsustainable. Consider the network peak as landscape value, and master the landscape value of single species, then we can combined it into a total ratio through these landscape value summation. This paper analyzed the various species landscape value.

\section{Conclusion}

The advantages of overall landscape model have its flexibility in gradual implement management, which can be applied to a part of the landscape. For example, it is used in strategic transfer, which is beneficial to the current agriculture practice. In addition, two or more landscape types can also be choosed in the same time, and we can focus on spatial continuity and ecological network.Besides,the application of landscape method is the basis of spatial planning, which is benefit for coastal landscape culture.

\section{References}

[1] PeiGen. The exploration and practice of urban design Based on the view of landscape protection . Journal of planning and design, 2011 (08):94-99.

[2] ZhuDongfeng Coastal development international experience and the enlightenment of the jiangsu province . International urban planning, 2009 (24):37-41.

[3] LiXiaohui. Landscape methods in wetland conservation and reasonable utilization of application. Wetland science and management, 2007 (3);53-56.

[4] Wang Lin. Nantong landscape pattern and landscape ecological planning research .Nanjing normal university press, 2006.

[5] LiuZhenren. Landscape cognition to huashan scenic spot planning and design influence landscape ecological analysis method application trial. Xi'an university of architecture \&technology press, 2012 (8):145-149.

[6] YuQing, ChenHaimu. Ecological network and green way .Beijing building industry press, 2011

[7] XiaYu, Zhang Hui. The landscape form of value, the research methods and rectification. Anhui agricultural science, 2010,(27):35-37

[8] HongWei. Landscape ecology in the application of wetland.Forestry research, 2010 (12):33-39.

[9] YangHuan. Research on urban green space landscape in Hangzhou.Beijing forestry university press, 2009.

[10] WuJianGuo. Landscape ecology -- pattern, process, scale and grade.Higher education press, 2002:664-668. 